\title{
Some quotients of chain products are symmetric chain orders
}

\author{
Dwight Duffus \\ Mathematics \& Computer Science Department \\ Emory University \\ Atlanta GA 30322, USA \\ dwight@mathcs. emory . edu \\ Jeremy McKibben-Sanders Kyle Thayer \\ Mathematics \& Computer Science Department \\ Emory University \\ Atlanta GA 30322, USA \\ \{jmckib2,kyle.thayer\}@gmail.com
}

Submitted: Oct 8, 2011; Accepted: Jun 12, 2012; Published: Jun 20, 2012

Mathematics Subject Classification: 06A07

\begin{abstract}
Canfield and Mason have conjectured that for all subgroups $G$ of the automorphism group of the Boolean lattice $B_{n}$ (which can be regarded as the symmetric group $S_{n}$ ) the quotient order $B_{n} / G$ is a symmetric chain order. We provide a straightforward proof of a generalization of a result of K. K. Jordan: namely, $B_{n} / G$ is an SCO whenever $G$ is generated by powers of disjoint cycles. In addition, the Boolean lattice $B_{n}$ can be replaced by any product of finite chains. The symmetric chain decompositions of Greene and Kleitman provide the basis for partitions of these quotients.
\end{abstract}

Keywords: symmetric chain decomposition, Boolean lattice, quotients

\section{Introduction}

There are several familiar notions of symmetry for the family of finite ranked partially ordered sets. This family can be defined in more general ways (see [9]), but for our purposes, all of our finite partially ordered sets $P$ have a minimum element $0_{P}$ and for all $x \in P$, all saturated chains $C \subseteq P$ with minimum element $0_{P}$ and maximum $x$ have the same length $r_{P}(x)=|C|-1$. Such $P$ are called ranked posets, $r=r_{P}$ is the rank function and $r(P)$, the maximum over all $r(x), x \in P$, is the rank of $P$. Note that a ranked ordered 
set satisfies the Jordan-Dedekind chain condition: for all $x \leqslant y$ in $P$, all saturated chains in the interval $[x, y]$ have the same length.

In a ranked order $P$ the chain $x_{1}<x_{2}<\cdots<x_{k}$ is a symmetric chain if it is saturated and if $r\left(x_{1}\right)+r\left(x_{k}\right)=r(P)$. A symmetric chain decomposition or $S C D$ of $P$ is a partition of $P$ into symmetric chains. If $P$ has an SCD, call $P$ a symmetric chain order, or an $S C O$. Here, we are concerned with ordered sets based on the Boolean lattice, denoted $B_{n}$, which is the power set of $[n]=\{1,2, \ldots, n\}$ ordered by containment. Clearly $B_{n}$ is a ranked poset, with $\emptyset$ being the minimum element, and $r(A)=|A|$ for all $A \subseteq[n]$. In fact, it is an SCO [2].

We are interested in ordered sets defined by actions of the automorphism group of $B_{n}$. It is well-known that this group is faithfully induced by the symmetric group $S_{n}$ of all permutations on the underlying set $[n]$, so we will refer to $S_{n}$ as the automorphism group of $B_{n}$. Given any subgroup $G$ of $S_{n}$, the quotient $B_{n} / G$ has as its elements the orbits in $B_{n}$ under $G$

$$
[A]=\{B \mid B=\sigma(A), \text { for some } \sigma \in G\}
$$

$A \in B_{n}$, ordered by

$$
[A] \leqslant[B] \Longleftrightarrow X \subseteq Y \text { for some } X \in[A] \text { and } Y \in[B] .
$$

In studying Venn diagrams, Griggs, Killian and Savage [12] explicitly constructed an SCD of the quotient $B_{n} / G$ for $n$ prime and given that $G$ is generated by a single $n$-cycle. They asked if this necklace poset is an SCO for arbitrary $n$. Canfield and Mason [3] made a much more general conjecture: for all subgroups $G$ of $S_{n}, B_{n} / G$ is a symmetric chain order.

Jordan [14] gave a positive answer to the question of Griggs, Killian and Savage, basing the SCD of the quotient on the explicit construction of an SCD in $B_{n}$ by Greene and Kleitman [10]. The construction in [14] requires an intermediate equivalence relation and some careful analysis. Here we provide a more direct proof of a generalization of Jordan's theorem by "pruning" the Greene-Kleitman SCD. More generally, we show that $B_{n} / G$ is an SCO provided that $G$ is generated by powers of disjoint cycles (see Theorem 1). We also provide a different proof that $B_{n} / G$ is an $\mathrm{SCO}$ when $G$ is a 2-element subgroup generated by a reflection, based on an SCD of $B_{\lfloor n / 2\rfloor}$.

The ordered sets $B_{n} / G$ do share several forms of symmetry or regularity with the Boolean lattice. An SCO $P$ is necessarily rank-symmetric, rank-unimodal, and strongly Sperner (see, for instance, [14] for definitions). A result of Stanley [17] shows that $B_{n} / G$ has these three properties for all subgroups $G$ of $S_{n}$. However, these three conditions are not sufficient to yield symmetric chain decompositions.

On the other hand, Griggs [11] showed that a ranked ordered set with the LYM property, rank-symmetry and rank-unimodality is an SCO. It is not the case that all SCOs have the LYM property (see [11] for examples), in fact, not all quotients $B_{n} / G$ have the LYM property. However, if $n$ is prime and $G$ is generated by an $n$-cycle, then $B_{n} / G$ does satisfy LYM, giving the Griggs, Killian and Savage result. Pouzet and Rosenberg [16] obtain Stanley's results and "local" families of symmetric chains for more general structures than the quotients $B_{n} / G$, but their results do not show that $B_{n} / G$ is an SCO. 


\section{The Main Results}

There are two results for quotients of $B_{n}$ by groups generated by powers of disjoint cycles and for a particular 2-element group. The third theorem concerns quotients of powers of a finite chain, with a corollary for products of chains of differing length.

Theorem 1. Let $G$ be a subgroup of $S_{n}$ generated by powers of disjoint cycles. Then the partially ordered set $B_{n} / G$ is a symmetric chain order.

The proof of Theorem 1 follows from this sequence of results. The new proof of Lemma 2 , which is a modest generalization of Jordan's result, is given in Section 3.

Lemma 2. Let $\sigma$ be an $n$-cycle in $S_{n}$ and let $H$ be a subgroup of the group generated by $\sigma$. Then $B_{n} / H$ is a symmetric chain order.

The following fact is well-known and can be proved by an argument much like the original proof in [2] that the divisor lattice of an integer is an SCO. (In [9], this is credited to Alekseev [1].)

Lemma 3. Let $P$ and $Q$ be partially ordered sets. If $P$ and $Q$ are symmetric chain orders then so is $P \times Q$.

In the following lemma, use this notation. Suppose that $\sigma_{j}(j=1,2, \ldots, t)$ are disjoint cycles in $S_{n}$ and that $\rho_{j}=\sigma_{j}^{r_{j}}$, for integers $r_{1}, r_{2}, \ldots, r_{t}$. Let $X_{j}$ be the subset of $[n]$ of elements moved by $\rho_{j}(j=1,2, \ldots, t)$, and let $X_{0}$ be all elements of $[n]$ fixed by all the $\rho_{j}$ 's. Let $B(X)$ denote the Boolean lattice of all subsets of a set $X$.

Lemma 4. Let $H_{j}$ be the subgroup of $S_{n}$ generated by $\rho_{j}(j=1,2, \ldots, t)$ and let $G$ be the subgroup generated by $\left\{\rho_{1}, \rho_{2}, \ldots, \rho_{t}\right\}$. Then

$$
B_{n} / G \cong B\left(X_{0}\right) \times B\left(X_{1}\right) / H_{1} \times \cdots \times B\left(X_{t}\right) / H_{t}
$$

Proof. For any $A \subseteq[n]$, let $[A]$ denote its equivalence class in $B_{n} / G$, and let $A_{j}=A \cap X_{j}$, $j=0,1, \ldots, t$. Define a map $\Phi$ on $B_{n} / G$ by $\Phi([A])=\left(A_{0},\left[A_{1}\right], \ldots\left[A_{t}\right]\right)$. From the definition of the ordering of the quotient, $[A] \leqslant[B]$ in $B_{n} / G$ if and only if there is some $\tau \in G$ such that $A \subseteq \tau(B)$. Then $\tau=\rho_{1}^{i_{1}} \rho_{2}^{i_{2}} \cdots \rho_{t}^{i_{t}}$ for nonnegative integers $i_{1}, i_{2}, \ldots, i_{t}$. The claimed isomorphism follows from this fact:

$$
A \subseteq \tau(B) \text { if and only if } A_{0} \subseteq B_{0} \text { and } A_{j} \subseteq \sigma^{i_{j}}\left(B_{j}\right) \text { for } j=1,2, \ldots, t .
$$

The following is actually a corollary of Theorem 1. Indeed, a proof based on an approach like that used in the proof of Theorem 1 - a greedy pruning of a Greene-Kleitman SCD - can be shown to provide a basis for the proof offered in Section 4. However, the proof in Section 4 provides some insight into the Greene-Kleitman SCD and may be of use for other choices for the group of permutations, such as the dihedral group, which is an appealing next case for study. 
Theorem 5. Let $G$ be a 2-element subgroup with non-unit element a product of disjoint transpositions. Then the partially ordered set $B_{n} / G$ is a symmetric chain order.

The last result concerns quotients defined by automorphism groups of products of chains. Given any partially ordered set $P$ and subgroup $G$ of its automorphism group Aut $(P)$, the quotient $P / G$ has elements the orbits $[x]$ on $P$ defined by $G$ with $[x] \leqslant[y]$ in $P / G$ if there are $x^{\prime} \in[x]$ and $y^{\prime} \in[y]$ such that $x^{\prime} \leqslant y^{\prime}$ in $P$. For any finite chain $C$, positive integer $m$ and $\alpha \in \operatorname{Aut}\left(C^{m}\right)$, there is some $\phi \in S_{m}$ such that

$$
\alpha\left(c_{1}, c_{2}, \ldots, c_{m}\right)=\left(c_{\phi^{-1}(1)}, c_{\phi^{-1}(2)}, \ldots, c_{\phi^{-1}(m)}\right), \text { for all }\left(c_{1}, c_{2}, \ldots, c_{m}\right) \in C^{m} .
$$

This follows easily from the action of the automorphisms on the covers of the minimum element of the chain product. (It is also a consequence of a result of Chang, Jónsson and Tarski [4], on the strict refinement property for product decompositions of partially ordered sets.) In particular, automorphism groups of powers of chains behave as those of the Boolean lattice and we can regard $\operatorname{Aut}\left(C^{m}\right)$ as the symmetric group $S_{m}$ acting on the coordinates of $C^{m}$.

Theorem 6. Let $C$ be a chain and let $K$ be a subgroup of $S_{m}$ generated by powers of disjoint cycles. Then $C^{m} / K$ is an $S C O$.

The proof, presented in Section 5, is a consequence of the proof of Lemma 2 and some observations on the Greene-Kleitman SCD. V. Dhand [5] has a new, very interesting result that is more general than the essential part of Theorem 6: if $P$ is any SCO then so is $P^{n} / \mathbb{Z}_{n}$. His arguments depend upon algebraic tools. P. Hersh and A. Schilling give a new proof of Jordan's result via an explicit combinatorial construction of an SCD in $B_{n} / \mathbb{Z}_{n}$ based on representation of the special linear group [13].

We note that Theorem 6 can be stated more generally for chain products. Let $P=$ $\prod_{i=1}^{n} C_{i}^{m_{i}}$ where $C_{j} \not C_{k}$ for $j \neq k$. It is easy to see that each automorphism of $P$ factors into an $n$-tuple from $\prod_{i=1}^{n} \operatorname{Aut}\left(C_{i}^{m_{i}}\right)$ and that each $\operatorname{Aut}\left(C_{i}^{m_{i}}\right) \cong S_{m_{i}}$. (For a much more general result based [4], see [6].) Thus, if $K$ is a subgroup of $\operatorname{Aut}(P)$ which also factors into a product of subgroups of $S_{m_{i}}$ of the form covered by Theorem 6 then, by Lemmas 3 and $4, P / K$ is an SCO. In particular, we have this consequence.

Corollary 7. Let $P$ be a product of chains and let $K$ be a subgroup of $\operatorname{Aut}(P)$ that is generated by powers of disjoint cycles. Then $P / K$ is an SCO.

We use Corollary 7 to deal with some cases where $K$ does not factor so nicely in [8].

\section{The Proof of Lemma 2}

We use the natural order $1<2<\ldots<n$ on $[n]$ and may assume that the $n$-cycle $\sigma$ is $(12 \cdots n)$. This is valid because any $n$-cycle $\rho$ is a conjugate of $(12 \cdots n)$ and for any subgroup $K$ of $S_{n}$ and any $\pi \in S_{n}, B_{n} / K \cong B_{n} / \pi^{-1} K \pi$ via $[A] \mapsto[\pi(A)]$. 
We first describe the procedure for obaining an SCD of $B_{n} / H$ based on the GreeneKleitman SCD of $B_{n}$ then verify that the procedure yields the claimed SCD.

Let $C_{1}, C_{2}, \ldots, C_{t}$, where $t=\left(\begin{array}{c}n \\ \lfloor n / 2\rfloor\end{array}\right)$, be the symmetric chains in the Greene-Kleitman decomposition, ordered by decreasing length. For all $A \in B_{n},[A]$ is the equivalence class containing $A$ in $B_{n} / H$ where $H$ is the subgroup of $S_{n}$ generated by $\rho=\sigma^{s}$.

Claim 1: There is a family $\mathcal{C}=\left\{C_{i_{1}}^{\prime}, C_{i_{2}}^{\prime}, \ldots C_{i_{m}}^{\prime}\right\}$, with $\left(i_{1}, i_{2}, \ldots, i_{m}\right)$ a subsequence of $(1,2, \ldots, t)$, that satisfies these conditions:

(3.1) for all $1 \leqslant j \leqslant m, C_{i_{j}}^{\prime} \subseteq C_{i_{j}}$ and is a symmetric chain in $B_{n}$;

(3.2) for all $1 \leqslant r<s \leqslant m$ and for all $A \in C_{i_{r}}^{\prime}, B \in C_{i_{s}}^{\prime}, A \notin[B]$; and,

(3.3) for all $[X]$ there is some $Y \in[X]$ such that $Y \in C_{i_{j}}^{\prime}$ for some $j$.

For $j=1,2, \ldots, m$, let $\widehat{C}_{j}=\left\{[A] \mid A \in C_{i_{j}}^{\prime}\right\}$. Then the chains $\widehat{C}_{1}, \widehat{C}_{2}, \ldots, \widehat{C}_{m}$ cover $B_{n} / H$ (by (3.3)), the sets are disjoint (by (3.2)), and form symmetric chains (by (3.1)). Thus, it is enough to verify Claim 1 in order to prove Lemma 2.

Several properties of the Greene-Kleitman SCD of $B_{n}$ are needed. For the most part, these are well-known - see, for instance, the descriptions in [9] and [14]. It is useful to regard members of $B_{n}$ both as subsets of $[n]$ and as binary sequences of length $n$, defined with respect to the natural order. (Indeed, one needs to fix an order to speak of the Greene-Kleitman SCD.) The SCD is obtained by a bracketing or pairing procedure that has several equivalent descriptions. Here are two that are useful to us. Let $A \subseteq[n]$.

(3.4) If $1 \notin A$ and $2 \in A$, pair 1 and 2 ; define $p_{A}(2)=1$. Suppose that we have considered $1,2, \ldots, k-1$. If $k \in A$ and there is some $j<k, j \notin A$ such that $j$ is unpaired, then let $p_{A}(k)$ be the maximum such $j$ and say $p_{A}(k)$ and $k$ are paired. Continue for all $k$ in $[n]$.

(3.5) For all $x \in A$ such that precisely half of the elements of the interval $[y, x]$ are members of $A$, for some $1 \leqslant y<x$, let $p_{A}(x)$ be the maximum such $y$.

Let $R(A)$ be the set of all $x$ for which $p_{A}(x)$ is defined, let $L(A)=\left\{p_{A}(x) \mid x \in R(A)\right\}$, and let $P(A)=L(A) \cup R(A)$. Now set

$$
f(A)=A \cup\{z\}, z=\min ([n]-(A \cup L(A))),
$$

if $[n]-(A \cup P(A)) \neq \emptyset$; otherwise $f(A)$ is undefined. Then this rule inverts $f$ :

$$
f^{-1}(B)=B-\{z\}, z=\max (B-R(B)) .
$$

Let $\mathcal{C}(A)=\left\{f^{k}(A) \mid k \in \mathbb{Z}\right\}$. As $A$ runs over all of $B_{n}$, the distinct $\mathcal{C}(A)$ 's provide the Greene-Kleitman SCD of $B_{n}$.

The following hold for all $A \in B_{n}$.

(3.6) For all $x \in R(A),\left[p_{A}(x), x\right] \subseteq P(A)$. 
(3.7) $\mathcal{C}(A)=\left\{X \in B_{n} \mid R(X)=R(A)\right\}$ and $p_{X}(a)=p_{A}(a)$ for all $X \in \mathcal{C}(A)$ and for all $a \in R(A)$.

(3.8) $\min (\mathcal{C}(A))=R(A), \max (\mathcal{C}(A))=[n]-L(A)$; in fact, $\mathcal{C}(A)$ is the chain $R(A) \subset R(A) \cup\left\{a_{1}\right\} \subset R(A) \cup\left\{a_{1}, a_{2}\right\} \subset \ldots \subset R(A) \cup\left\{a_{1}, a_{2}, \ldots a_{t}\right\}=[n]-L(A)$, where $[n]-(R(A) \cup L(A))=\left\{a_{1}<a_{2}<\ldots<a_{t}\right\}$.

The following two lemmas provide properties of this SCD that substantiate Claim 1. Given a symmetric chain $C$ in $B_{n}$ and $X \in C$ with $|X| \leqslant\lfloor n / 2\rfloor$, let $X^{*}$ be the member of $C$ with $\left|X^{*}\right|=n-|X|$.

Lemma 8. For $i=1,2, \ldots, t$ and for all $X \in C_{i}$ with $|X| \leqslant\lfloor n / 2\rfloor,(\sigma(X))^{*}=\sigma\left(X^{*}\right)$. Thus $\left(\sigma^{j}(X)\right)^{*}=\sigma^{j}\left(X^{*}\right)$ for all integers $j$, so $(\rho(X))^{*}=\rho\left(X^{*}\right)$ for all $\rho \in H$.

A special case of the preceding lemma is in [7]. Since this reference is a technical report and the result does not appear to be available in the literature, we prove this below. (We note that for $n$ odd, the middle edges of the Greene-Kleitman SCD are the edges of the well-known lexicographic matching in the middle two levels graph. The invariance of this matching under rotation is a special case of a result of Kierstead and Trotter [15] on what they call $i$-lexical matchings.)

Here is the second property of the Greene-Kleitman SCD that we require.

Lemma 9. Let $w \in\{1,2, \ldots, t\}$, and let $A \in C_{w}$ with $|A| \leqslant\lfloor n / 2\rfloor$. Suppose that there is some $B \in[A]$ such that $B \in C_{j}$ for some $j<w$. Then there is some $k<w$ and $D \in C_{k}$ such that $D \in\left[f^{-1}(A)\right]$, provided that $f^{-1}(A)$ is defined.

To prove Claim 1 from these facts, define $\mathcal{C}$ inductively.

First, let $i_{1}=1$ and $C_{i_{1}}^{\prime}=C_{1}$. Suppose that $C_{i_{1}}^{\prime}, C_{i_{2}}^{\prime}, \ldots, C_{i_{k}}^{\prime}$ are defined. If there exists $i \in\left\{i_{k}+1, \ldots, t\right\}$ such that for some $X \in C_{i}$,

$$
[X] \cap\left(\bigcup_{j=1}^{k} C_{i_{j}}^{\prime}\right)=\emptyset
$$

let $i_{k+1}$ be the least such $i$ and let

$$
C_{i_{k+1}}^{\prime}=\left\{Y \in C_{i_{k+1}} \mid[Y] \cap\left(\bigcup_{j=1}^{k} C_{i_{j}}^{\prime}\right)=\emptyset\right\} .
$$

If there is no such $i$ then $m=k$, that is, $C_{i_{m}}^{\prime}$ is the last chain required by Claim 1 and the procedure is complete.

If $Y \in C_{i_{k+1}}^{\prime}$, with $|Y| \leqslant\lfloor n / 2\rfloor$ then $Y^{*} \in C_{i_{k+1}}^{\prime}$, by the dual of Lemma 8. Also, if $Z \in C_{i_{k+1}}$ and $Y \subseteq Z \subseteq Y^{*}$ where $Y \in C_{i_{k+1}}^{\prime}$ then $Z \in C_{i_{k+1}}^{\prime}$ by Lemma 9 and Lemma 8. Thus, $C_{i_{k+1}}^{\prime}$ is symmetric in $B_{n}$ and (3.1) holds. Equation (2) verifies (3.2); (3.3) follows from (1) and (2). 
Proof of Lemma 8. The proof is divided into cases depending upon which of $R(X) \subseteq$ $X \subseteq X^{*}$ contain $n$. It is not possible that $n \in X-R(X)$, because $|X| \leqslant\lfloor n / 2\rfloor$ means that for some $y<n$ precisely half the elements of $[y, n]$ are in $X$, and, hence, $n \in R(X)$ by (3.5). Consequently, there are three cases. In each case, we show that

$$
R\left(\sigma\left(X^{*}\right)\right)=R(\sigma(X))
$$

apply (3.7) to see that $\sigma\left(X^{*}\right)$ and $(\sigma(X))^{*}$ are both members of $\mathcal{C}(\sigma(X))$, and conclude that $\sigma\left(X^{*}\right)=(\sigma(X))^{*}$ since these sets both have cardinality $n-|\sigma(X)|$.

Case 1: $n \notin X^{*}$

Since $n \in[n]-L(X)=\max (\mathcal{C}(X))$ and $n \notin X^{*}, X \neq \min (\mathcal{C}(X))=R(X)$. Thus, there exists $y=\min (X-R(X))$. If $y=1$ then $p_{\sigma(X)}(2)=1=p_{\sigma\left(X^{*}\right)}(2)$. For each $z \in R(X), \sigma(z)=z+1 \in R(\sigma(X))$ and each $z+1 \in R(\sigma(X))$ has $z \in R(X)$ apart from $z+1=2$. Thus,

$$
\begin{array}{rlrl}
R(\sigma(X)) & =\sigma(R(X) \cup\{2\} & & \text { since } p_{\sigma(X)}(2)=1, \\
& =\sigma\left(R\left(X^{*}\right)\right) \cup\{2\} & \text { by }(3.7), \\
& =R\left(\sigma\left(X^{*}\right)\right) & & \text { since } p_{\sigma\left(X^{*}\right)}(2)=1 .
\end{array}
$$

If $y>1$ we claim that $[1, y-1] \subseteq P(X)$. Note that $y-1 \in X$ as otherwise $y \in R(X)$ with $p_{X}(y)=y-1$, contradicting the choice of $y$. By the minimality of $y, y-1 \in R(X)$ and, by (3.7), $\left[p_{X}(y-1), y-1\right] \subseteq P(X)$. Continue in the same manner, with $p_{X}(y-1)-1$ in place of $y$, and thereby verify the claim that $[1, y-1] \subseteq P(X)$. The argument is just about the same as when $y=1$ except we use the fact that $[1, y-1] \subseteq P(X)$ and $1 \notin X$, so, $p_{\sigma(X)}(y+1)=1$ :

$$
R\left(\sigma\left(X^{*}\right)\right)=\sigma\left(R\left(X^{*}\right)\right) \cup\{y+1\}=\sigma(R(X)) \cup\{y+1\}=R(\sigma(X)) .
$$

Case 2: $n \in R(X)$

Every element of $R(\sigma(X))$ is in $\sigma(R(X))$ and every element of $\sigma(R(X))$, except for 1 , is in $R(\sigma(X)$. Thus,

$$
R(\sigma(X))=\sigma(R(X))-\{1\}=\sigma\left(R\left(X^{*}\right)\right)-\{1\}=R\left(\sigma\left(X^{*}\right)\right) .
$$

Case 3: $n \in X^{*}-X$

Since $n \in X^{*}-X$, (3.8) shows that $X^{*}=\max (\mathcal{C}(X))=[n]-L(X)$ and, thus, $X=\min (\mathcal{C}(X))=R(X)$.

If $z+1 \in R(\sigma(X))$ then $z \in X=R(X)$, so $R(\sigma(X)) \subseteq \sigma(R(X))$. Conversely, $1 \notin \sigma(R(X))$, and any $z+1 \in \sigma(R(X))$ is obviously a member of $R(\sigma(X))$. Thus, $R(\sigma(X))=\sigma(R(X))$. Similarly, since $n \notin R(X)$, it follows that $R\left(\sigma\left(X^{*}\right)\right)=\sigma\left(R\left(X^{*}\right)\right)$. Hence, $R\left(\sigma\left(X^{*}\right)\right)=R(\sigma(X))$.

Since $(\sigma(X))^{*}=\sigma\left(X^{*}\right)$ for all $X$ with $|X| \leqslant\lfloor n / 2\rfloor$, we can apply induction on $j$ to conclude that $\left(\sigma^{j}(X)\right)^{*}=\sigma^{j}\left(X^{*}\right)$ :

$$
\left(\sigma^{j}(X)\right)^{*}=\left(\sigma\left(\sigma^{j-1}(X)\right)\right)^{*}=\sigma\left(\left(\sigma^{j-1}(X)\right)^{*}\right)=\sigma\left(\sigma^{j-1}\left(X^{*}\right)\right)=\sigma^{j}\left(X^{*}\right) .
$$


Proof of Lemma 9. As before, let $C_{1}, C_{2}, \ldots, C_{t}$, where $t=\left(\begin{array}{c}n \\ \lfloor n / 2\rfloor\end{array}\right)$, be the symmetric chains in the Greene-Kleitman decomposition, ordered by decreasing length, and let $\sigma=$ $(12 \cdots n)$. Let $A \in C_{w}$ with $|A| \leqslant\left\lfloor\frac{n}{2}\right\rfloor$. Suppose that there exists a $j<w$ such that $B \in C_{j}$ and $B \in[A]$. Hence there is an integer $r$ such that $B=\sigma^{r}(A)$.

Assume that $f^{-1}(A)$ is defined. We show that there is a $k<w$ such that $D \in C_{k}$ and $D \in\left[f^{-1}(A)\right]$. Since $f^{-1}(A)$ is defined, $A-R(A) \neq \emptyset$. Let $y=\max (A-R(A))$. We may assume that $-(y-1) \leqslant r \leqslant n-y, r \neq 0$. We consider two cases:

Case 1: $y+r \in R(B)$

Then $r>0$ since otherwise $y$ would also be paired in $A$, contrary to its choice. Each $z \in B$ with $y+r<z$ must be in $R(B)$ since $y<z-r$ so, by the choice of $y, z-r \in R(A)$. Now consider the binary sequence $\sigma^{r}\left(f^{-1}(A)\right)$ contained in some chain $C_{k}$. Recall that $f^{-1}(A)=A-\{y\}$ and note that $\sigma^{r}\left(f^{-1}(A)\right)=B-\{y+r\}$. It follows from this that $\sigma^{r}\left(f^{-1}(A)\right)$ must have one fewer pairs than $B$, since $y+r$ will be unpaired in $\sigma^{r}\left(f^{-1}(A)\right)$ while $y+r$ is paired in $B$, and there are no other differences in the pairings. By (3.8), $\left|C_{k}\right|>\left|C_{j}\right|$, so $k<j<w$, as desired.

Case 2: $y+r \in B-R(B)$

If $y+r=\max (B-R(B))$ then we are done, since then we have $f^{-1}(B)=\sigma^{r}\left(f^{-1}(A)\right)$. So suppose instead $z=\max (B-R(B))$ where $y+r<z$. If $r>0$ then $z-r \in(A-R(A))$, contrary to the choice of $y$. Thus $r<0$. If $z-r \leqslant n$ then $z-r$ would be an unpaired element of $A$, since $y<z-r$ remains unpaired in $A$. This would contradict the choice of $y$. Thus $n<z-r$.

We now prove that for some $p, \sigma^{p}(B)$ is the maximum element of its chain, and its chain is not a singleton. This will contradict the fact that $|A| \leqslant\left\lceil\frac{n}{2}\right\rceil$, since $\left|\sigma^{p}(B)\right|=|A|$.

Since $\sigma^{-r}(B)=A,-r>0$, we obtain $A$ from $B$ by applying $\sigma-r$ times. Since $n<z-r$ there is some $p$ such that $\sigma^{p}(z)=n$. Let $X=\sigma^{p}(B)$. Then, $\sigma^{p}(z) \in X-R(X)$. Because $n \in X-R(X), X=[n]-L(X)$. By (3.8), $X$ is the maximum element of its chain. The chain containing $X$ is not a singleton, since $X-R(X) \neq \emptyset$.

\section{The proof of Theorem 5}

Let $\rho=\left(i_{1} j_{i}\right)\left(i_{2} j_{2}\right) \cdots\left(i_{k} j_{k}\right)$, where the transpositions are pairwise disjoint, let $X=$ $\bigcup_{r=1}^{k}\left\{i_{r}, j_{r}\right\}$, and let $G=\{1, \rho\}$. Then

$$
B_{n} / G \cong B(X) / G \times B([n]-X)
$$

via the mapping $[A] \mapsto([A \cap X], A-X)$ for all $A \subseteq[n]$. By Lemma 3, we may assume that $n$ is even and that $n=2 k$. Using the remark about conjugation at the beginning of Section 3, we may assume that $\rho=(12 k)(22 k-1) \cdots(k k+1)$. As noted in the introduction, $G$ can be generated by a power of a $2 k$-cycle, so Theorem 1 applies. (In fact, $\rho=\tau^{-1} \sigma \tau$ where $\sigma=(12 \cdots 2 k)$ and $\tau=(k+12 k)(k+22 k-1) \cdots$. ) And, as we shall see, its proof method can be adapted to give the proof we offer here. However, the argument below might help with the most interesting open case, namely, showing that $B_{n} / D_{2 n}$ is an SCO for the dihedral group $D_{2 n}$. 
Regard each $A \in B_{2 k}$ as a concatenated pair of binary strings of length $k$. That is, $A=\mathbf{b}_{1} \mathbf{b}_{2}^{r}$, where $\mathbf{b}_{1}, \mathbf{b}_{2} \in\{0,1\}^{k}$ and $\mathbf{b}^{r}$ is the reverse of the binary $k$-sequence $\mathbf{b}$. Then the equivalence classes in $B_{n} / G$ are the sets $\left\{\mathbf{b}_{1} \mathbf{b}_{2}^{r}, \mathbf{b}_{2} \mathbf{b}_{1}^{r}\right\}$; these sets have 2 elements except in the case that $\mathbf{b}_{1}=\mathbf{b}_{2}$.

Let $C_{1}, C_{2}, \ldots, C_{t}$, where $t=\left(\begin{array}{c}k \\ \lfloor k / 2\rfloor\end{array}\right)$, be any symmetric chain decomposition of $B_{k}$, ordered by decreasing length. We define a total ordering $\preccurlyeq$ on $B_{k}=\{0,1\}^{k}$ as follows:

$$
\mathbf{b}_{r} \preccurlyeq \mathbf{b}_{s} \text { if } \mathbf{b}_{r} \in C_{i}, \mathbf{b}_{s} \in C_{j}, i<j \text {, or if } \mathbf{b}_{r} \subseteq \mathbf{b}_{s} \text { in } C_{i} \text { for some } i \text {. }
$$

For $1 \leqslant i<j \leqslant t$, let $P_{i j}=C_{i} \times C_{j}$, with the coordinate-wise ordering induced by the containment order on $B_{k}$, for each $i=1,2, \ldots, t$, let

$$
P_{i i}=\left\{\left(\mathbf{b}_{r}, \mathbf{b}_{s}\right) \in C_{i} \times C_{i} \mid \mathbf{b}_{r} \subseteq \mathbf{b}_{s}\right\},
$$

ordered coordinate-wise, and let

$$
P=\bigcup_{1 \leqslant i \leqslant j \leqslant t} P_{i j}
$$

again, ordered coordinate-wise. Thus, $P$ is a subset of $B_{2 k}$ with the exactly the ordering inherited from the Boolean lattice.

In fact, with $r_{P}$ and $r_{B_{k}}$ as the rank functions in $P$ and $B_{k}$, respectively, then for $1 \leqslant i \leqslant j \leqslant t$ and with $r_{B_{k}}\left(\min C_{i}\right)=r_{i}$, and $r_{B_{k}}\left(\max C_{i}\right)=k-r_{i}$,

$$
r_{P}\left(\min P_{i j}\right)=r_{i}+r_{j}, r_{P}\left(\max P_{i j}\right)=2 k-\left(r_{i}+r_{j}\right) \text {, and } l\left(P_{i j}\right)=2 k-2\left(r_{i}+r_{j}\right) .
$$

We see that each $P_{i j}$ is a symmetric subset of $B_{2 k}$ in which the covering relation is preserved, that is, $\left(\mathbf{b}_{p}, \mathbf{b}_{q}\right)$ is covered by $\left(\mathbf{b}_{u}, \mathbf{b}_{v}\right)$ in some $P_{i j}$ if and only if $\mathbf{b}_{p} \mathbf{b}_{q}^{r}$ is covered by $\mathbf{b}_{u} \mathbf{b}_{v}^{r}$ in $B_{2 k}$.

Consider the map $\phi$ of $P$ to $B_{2 k} / G$ defined by $\phi\left(\left(\mathbf{b}_{1}, \mathbf{b}_{2}\right)\right)=\left\{\mathbf{b}_{1} \mathbf{b}_{2}^{r}, \mathbf{b}_{2} \mathbf{b}_{1}^{r}\right\}$. Since $\mathbf{b}_{1} \preccurlyeq \mathbf{b}_{2}$ for all $\left(\mathbf{b}_{1}, \mathbf{b}_{2}\right) \in P, \phi$ is injective. It is obviously a surjection. It is also order-preserving: if $\left(\mathbf{b}_{p}, \mathbf{b}_{q}\right) \leqslant\left(\mathbf{b}_{u}, \mathbf{b}_{v}\right)$ in $P$ then $\mathbf{b}_{p} \mathbf{b}_{q}^{r} \leqslant \mathbf{b}_{u} \mathbf{b}_{v}^{r}$ in $B_{2 k}$.

Since the rank of an equivalence class in $B_{2 k} / G$ is the rank of its members in $B_{2 k}$, it follows that a symmetric chain in $P$ is a symmetric chain in $B_{2 k} / G$. Thus, it is enough to proof the following.

Claim 2: $P$ has a symmetric chain decomposition.

Since $P$ is partitioned by $P_{i j}, 1 \leqslant i \leqslant j \leqslant t$, each of which preserve the covering relation in $P$, it is enough to prove that each $P_{i j}$ has a partition into chains, each of which is symmetric in $P$.

For $1 \leqslant i<j \leqslant t, P_{i j}=C_{i} \times C_{j}$ is a cover-preserving subset of $P$, with minimum element at level $r_{i}+r_{j}$ and maximum element at level $2 k-\left(r_{i}+r_{l}\right)$ in $P$, a partially ordered set of length $2 k$. Then the "standard" symmetric partition of a product of two chains (the original partition in [2]) provides symmetric chains in $P$.

For $i=1,2, \ldots, t, P_{i i}=\left\{\left(\mathbf{b}_{r}, \mathbf{b}_{s}\right) \in C_{i} \times C_{i} \mid \mathbf{b}_{r} \subseteq \mathbf{b}_{s}\right\}$, where $C_{i}$ is the chain of binary strings $\mathbf{b}_{r_{i}} \subset \mathbf{b}_{r_{i}+1} \subset \cdots \subset \mathbf{b}_{k-r_{i}}$, where $r_{B_{k}}\left(\mathbf{b}_{s}\right)=s$ in $B_{k}$, that is, is an $s$-element set, 
for $s=r_{i}, r_{i}+1, \ldots, k-r_{i}$. Then $P_{i i}$ is an interval in $P$ with minimum element at level $2 r_{i}$ and maximum element at level $2 k-2 r_{i}$ in $P$. Also,

$$
\left(\mathbf{b}_{r_{i}}, \mathbf{b}_{r_{i}}\right)<\left(\mathbf{b}_{r_{i}}, \mathbf{b}_{r_{i}+1}\right)<\cdots<\left(\mathbf{b}_{r_{i}}, \mathbf{b}_{k-r_{i}}\right)<\left(\mathbf{b}_{r_{i}+1}, \mathbf{b}_{k-r_{i}}\right)<\cdots<\left(\mathbf{b}_{k-r_{i}}, \mathbf{b}_{k-r_{i}}\right)
$$

is a symmetric chain in $P$ and $P_{i i}-C$ is a cover-preserving subset of $P$, isomorphic to the product of two chains, with minimum element $\left(\mathbf{b}_{r_{i}+1}, \mathbf{b}_{r_{i}+1}\right)$ at level $2 r_{i}+2$ and maximum element $\left(\mathbf{b}_{k-r_{i}-1}, \mathbf{b}_{k-r_{i}-1}\right)$ at level $2 k-2 r_{i}-2$. By induction, we have a decomposition of $P_{i i}$ by chains symmetric in $P$. This verifies Claim 2 and completes the proof of Theorem 5 .

\section{The proof of Theorem 6}

With Lemmas 3 and 4 , it is enough to prove the result for $K$ generated by a single $m$ cycle. We assume that $C$ is the $k$-element chain $00 \ldots 0,10 \ldots 0,11 \ldots 0,111 \ldots 1$ in the Boolean lattice $B_{k-1}$. Let $n=(k-1) m$. Then $C^{m}$ is the sublattice of $B_{n}$ consisting of all binary sequences of length $n$ of the form

$$
\mathbf{b}=\mathbf{b}_{\mathbf{1}} \mathbf{b}_{\mathbf{2}} \ldots \mathbf{b}_{\mathbf{m}} \text {, where each } \mathbf{b}_{i} \in C .
$$

That is, the elements of $C^{m}$ are exactly those $n$-sequences which are $m(k-1)$-sequences, each comprised of 1's followed by 0's.

Let $C_{1}, C_{2}, \ldots, C_{t}$, where $t=\left(\begin{array}{c}n \\ \lfloor n / 2\rfloor\end{array}\right)$, be the symmetric chains in the Greene-Kleitman SCD of $B_{n}$, ordered by decreasing length, as in Section 3. We claim that for each $j$, $C_{j} \subseteq C^{m}$ or $C_{j} \cap C^{m}=\emptyset$.

Suppose that $\mathbf{b}=\mathbf{b}_{\mathbf{1}} \mathbf{b}_{\mathbf{2}} \ldots \mathbf{b}_{\mathbf{m}} \in C_{j} \cap C^{m}$. With the notation in (3.6) - (3.8), and applying these to $A=\mathbf{b}$, we can see that $i^{\text {th }}$ entry $b_{i}$ of $\mathbf{b}$ is determined as follows:

$$
b_{i}= \begin{cases}0 & i \in L(\mathbf{b}) \cup\left\{a_{r}, a_{r+1}, \ldots, a_{t}\right\} \\ 1 & i \in R(\mathbf{b}) \cup\left\{a_{1}, a_{2}, \ldots, a_{r-1}\right\} .\end{cases}
$$

for some $r$.

If $\mathbf{b}$ is not the maximum element of $C_{j}$ then its successor $\mathbf{b}^{\prime}$ is obtained by changing the 0 in position $a_{r}$ to a 1 . Either $a_{r}=1$ or the entry in $\mathbf{b}$ in position $a_{r}-1$ is a 1 , by (3.6). Thus, $\mathbf{b}^{\prime}$ consists of $m(k-1)$-sequences of 1 's followed by 0 's and, so, belongs to $C_{j} \cap C^{m}$. If $\mathbf{b}$ is not the minimum element of $C_{j}$ then its predecessor $\mathbf{b}^{\prime \prime}$ is obtained by changing the 1 in position $a_{r-1}$ to a 0 . Either $a_{r-1}=n$ or the entry in $\mathbf{b}$ in position $a_{r-1}+1$ is a 0 , by (3.6). Again $\mathbf{b}^{\prime \prime}$ consists of $m(k-1)$-sequences of 1 's followed by 0's and, so, belongs to $C_{j} \cap C^{m}$. Hence, if $C_{j} \cap C^{m} \neq \emptyset$ then $C_{j} \subseteq C^{m}$.

Let $K=\left\langle\phi^{r}\right\rangle$ where we may assume that $\phi=(12 \cdots m)$. We need an SCD for $C^{m} / K$. We know that $C^{m}$ is a sublattice of $B_{n}$, as noted above, and that $\phi^{r}=\sigma^{(k-1) r} \mid C^{m}$ where $\sigma=(12 \cdots n) \in S_{n}$. As in the proof of Lemma 2, Claim 1 gives an SCD $\widehat{C}_{j}=C_{i_{j}}^{\prime}$,

$j=1,2, \ldots, m$, of $B_{n} / H$ where $H=\left\langle\sigma^{(k-1) r}\right\rangle$. Thus, the subfamily of those $\widehat{C}_{j}, j=$ $1,2, \ldots, m$, such that $\widehat{C}_{j} \subseteq C^{m}$ is an SCD for $C^{m} / K$. 


\section{References}

[1] V. B. Alekseev. Use of symmetry in finding the width of partially ordered sets (Russian). Diskret. Analiz., 26: 20 - 35, 1974.

[2] N. G. de Bruijn, C. Tengbergen and D. Kruyswijk. On the set of divisors of a number. Nieuw Arch. Wiskd., 23: 191 - 193, 1951.

[3] E. R. Canfield and S. Mason. When is a quotient of the Boolean lattice a symmetric chain order? Preprint, 2006.

[4] C. C. Chang, B. Jónsson and A. Tarski. Refinement properties for relational structures. Fund. Math., 55: 249 - 281, 1964.

[5] V. Dhand. Symmetric chain decomposition of necklace posets. Electron. J. Combin., 19: P26, 2012

[6] D. Duffus. Automorphism and products of ordered sets. Algebra Universalis, 19: 366 - 369, 1984.

[7] D. Duffus, P. Hanlon and R. Roth. Matchings and hamiltonian cycles in some families of symmetric graphs. Emory University Technical Reports, 1986.

[8] D. Duffus and K. Thayer, Quotients of chain products and symmetric chain decompositions. Preprint, 2012.

[9] K. Engel. Sperner Theory. Cambridge University Press, 1997.

[10] C. Greene and D. J. Kleitman. Strong versions of Sperner's theorem. J. Combinatorial Theory A, 20: 80 - 88, 1976.

[11] J. R. Griggs. Sufficient conditions for a symmetric chain order. SIAM J. Appl. Math. 32: 807 - 809, 1977.

[12] J. R. Griggs, C. E. Killian and C. D. Savage. Venn diagrams and symmetric chain decompositions in the Boolean lattice. Electron. J. Combin., 11: R2, 2004.

[13] P. Hersh and A. Schilling. Symmetric chain decomposition for cyclic quotients of Boolean algebras and relation to cyclic crystals. Internat. Math. Res. Notices doi:10.1093/imrn/rnr254

[14] K. K. Jordan. The necklace poset is a symmetric chain order. J. Combinatorial Theory A 117: 625 - 641, 2010.

[15] H. Kierstead and W. T. Trotter. Explicit matchings in the middle levels of the Boolean lattice. Order 5: 163 - 171, 1988.

[16] M. Pouzet and I. G. Rosenberg. Sperner properties for groups and relations. Europ. J. Combinatorics 7: 349 - 370, 1986.

[17] R. P. Stanley. Quotients of Peck posets. Order, 1: 29 - 34, 1984. 